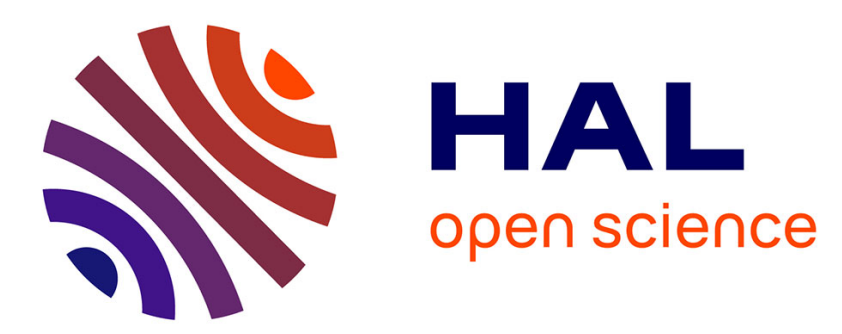

\title{
Robust design optimization with an uncertain model of a nonlinear vibro-impact electro-mechanical system
}

\author{
R. Lima, Christian Soize, R. Sampaio
}

\section{To cite this version:}

R. Lima, Christian Soize, R. Sampaio. Robust design optimization with an uncertain model of a nonlinear vibro-impact electro-mechanical system. Communications in Nonlinear Science and Numerical Simulation, 2015, 23 (1-3), pp.263-273. 10.1016/j.cnsns.2014.11.014 . hal-01137739

\author{
HAL Id: hal-01137739 \\ https://hal.science/hal-01137739
}

Submitted on 31 Mar 2015

HAL is a multi-disciplinary open access archive for the deposit and dissemination of scientific research documents, whether they are published or not. The documents may come from teaching and research institutions in France or abroad, or from public or private research centers.
L'archive ouverte pluridisciplinaire HAL, est destinée au dépôt et à la diffusion de documents scientifiques de niveau recherche, publiés ou non, émanant des établissements d'enseignement et de recherche français ou étrangers, des laboratoires publics ou privés. 


\title{
Robust design optimization with an uncertain model of a nonlinear vibro-impact electro-mechanical system
}

\author{
Roberta Lima, ${ }^{* a, b}$, Christian Soize ${ }^{\mathrm{a}}$, Rubens Sampaio ${ }^{\mathrm{b}}$ \\ ${ }^{a}$ Laboratoire de Modelisation et Simulation Multi Echelle, Equipe de Mecanique, \\ Paris-Est, 5, Boulevard Descartes, 77454, Marne-la-Vallee, Cedex 2, France \\ ${ }^{b}$ Department of Mechanical Engineering, PUC-Rio, Rua Marquês de Sao Vicente, 225, \\ Gávea, 22453-900, RJ, Brazil
}

\begin{abstract}
In this paper, the robust design with an uncertain model of a vibro-impact electro-mechanical system is done. The electro-mechanical system is composed of a cart, whose motion is excited by a DC motor (motor with continuous current), and an embarked hammer into this cart. The hammer is connected to the cart by a nonlinear spring component and by a linear damper, so that a relative motion exists between them. A linear flexible barrier, placed outside of the cart, constrains the hammer movements. Due to the relative movement between the hammer and the barrier, impacts can occur between these two elements. The developed model of the system takes into account the influence of the DC motor in the dynamic behavior of the system. Some system parameters are uncertain, such as the stiffness and the damping coefficients of the flexible barrier. The objective of the paper is to perform an optimization of this electro-mechanical system with respect to design parameters (spring component, and barrier $g$ ) in order to maximize the impact power under the constraint that the electric power consumed by the DC motor is lower than a maximum value. This optimization is formulated in the framework of robust design due to the presence of uncertainties in the model. The set of nonlinear equations are presented, and an adapted time domain solver is developed. The stochastic nonlinear constrained design
\end{abstract}

\footnotetext{
*Corresponding author

Email addresses: roberta.dequeirozlima@univ-paris-est.fr (Roberta Lima), christian.soize@univ-paris-est.fr (Christian Soize), rsampaio@puc-rio.br (Rubens Sampaio)
}

Preprint published to Communications in Nonlinear Science and Numerical Simulation 23(1-3) 2015 
optimization problem is solved for different levels of uncertainties, and also for the deterministic case.

Key words: electro-mechanical systems, vibro-impact, robust design optimization, nonlinear dynamics.

\section{Introduction}

Electro-mechanical systems are common in actual technologies, and their design is of a great interest in many areas. Many works have been done in this topic, as [1, 2, 3], trying to characterize the mutual interaction between electrical and mechanical parts. This interaction leads us to analyze very interesting nonlinear dynamical systems (see for instance $[4,5,6,7,8,9,10]$ ) , in which the nonlinearities vary with the coupling conditions, and also affects the two most important variables used to evaluate the performance of electro-mechanical systems, related to the power consumed by the electrical part, and the power used into the movement of the mechanical part. As the mutual interaction between electrical and mechanical parts affects the two powers used to evaluate the system performance, the coupling effects must be analyzed in the design optimization problem for electro-mechanical systems. The present work deals with the robust design optimization of a vibro-impact electric-mechanical system in order to improve its performance. The electrical part of the system is a DC motor, and the mechanical part is a vibro-impact system. It should be noted that, in [11], the equations and the numerical integration were presented for a similar electric-mechanical system for which the embarked mass was replaced by a pendulum and for which there was no impact. This first work has allowed the electro-mechanical coupling to be analyzed as a function of the mass of the mechanical system. The analysis of vibro-impact systems is not a new subject, and is frequently encountered in technical applications of mechanisms. The interest of analyzing the optimization of their performance is reflected by the increasing amount of research in this area (see for instance $[12,13,14]$, and also the book by Ibrahim [15], which is completely devoted to this problem). Besides the theoretical research in vibro-impact dynamics, the applications have also been developed, such as percussive actions in rotary drilling, vibration hammer, impact damper, and gears. The vibro-impact dynamics appears also in several other situations, as for example in earthquakes, where the interest is the seismic mitigation [16]. The vibro-impact dynamics can be affected by 
many factors, and the analysis of vibro-impact dynamical systems requires to taken into account uncertainties in the computational models that are used (see for instance [17]).

The objective of the paper is to perform an optimization of this electromechanical system with respect to design parameters that are the stiffness of the spring component, and the gap, $g$, of the barrier. The optimization consists in maximizing the impact power under the constraint that the electric power consumed by the DC motor is lower than a maximum value. This optimization is formulated in the framework of robust design due to the presence of uncertainties in the computational nonlinear dynamics model of the electro-mechanical system.

This paper is organized as follows. In Section 2, without introducing any simplifying hypotheses, the equations are constructed. The initial value problem is presented for the vibro-impact electro-mechanical system. In Section 3 we define the variables of interest for the design optimization. A sensitivity analysis with respect the system parameters is performed numerically in Section 4, in order to define the most sensitive parameters, and then to define what will be the design parameters. The construction of the probabilistic models of the uncertain parameters, and the formulation of the robust design optimization problem are given in Sections 5 and 6. The numerical results of the robust design optimization problem are presented in Section 7.

\section{Dynamic of the coupled system}

First, the elements (motor, cart, hammer, and barrier) of the electromechanical system are presented. The coupling between the motor and the mechanical system is described, and the time-evolution coupled problem is mathematically formulated as an initial-value problem.

\subsection{Electrical system: DC motor}

The mathematical modeling of DC motors is based on the Kirchhoff law [18]. It is written as

$$
\begin{aligned}
& l \dot{c}(t)+r c(t)+k_{e} \dot{\alpha}(t)=\nu, \\
& j_{m} \ddot{\alpha}(t)+b_{m} \dot{\alpha}(t)-k_{e} c(t)=-\tau(t),
\end{aligned}
$$

where $t$ is time, $\nu$ is the source voltage, $c$ is the electric current, $\dot{\alpha}$ is the angular speed of the motor, $l$ is the electric inductance, $j_{m}$ is the inertia 


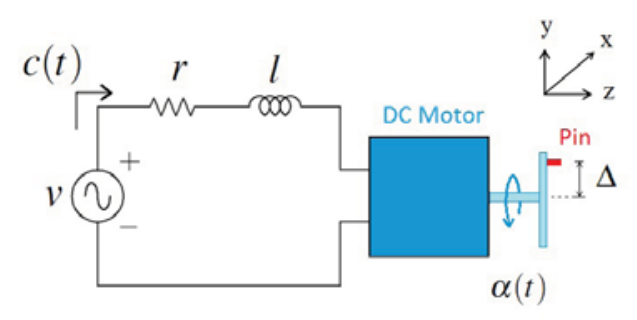

Figure 1: Sketch of the DC motor.

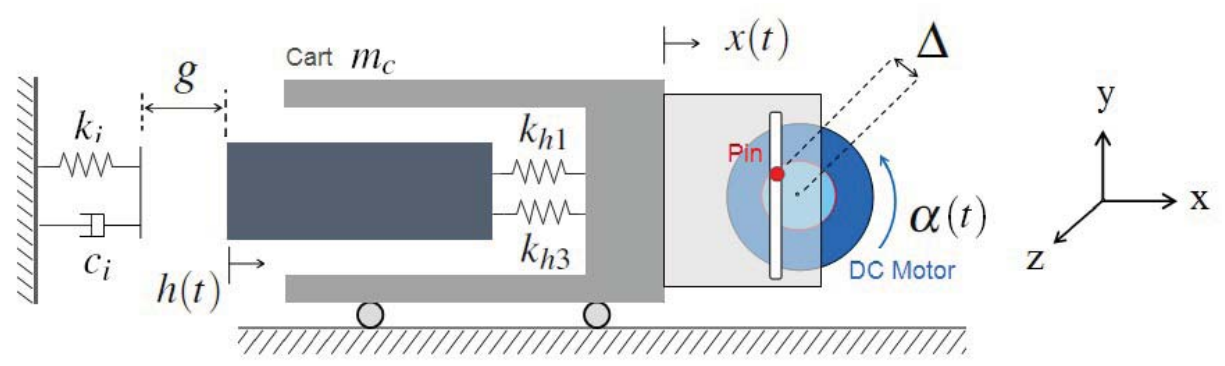

Figure 2: Motor-cart-hammer coupled system. The nonlinear component spring is drawn as a linear spring with constant $k_{h 1}$ and a nonlinear cubic spring with constant $k_{h 3}$.

moment of the motor, $b_{m}$ is the damping ratio in the transmission of the torque generated by the motor to drive the coupled mechanical system, $k_{e}$ is the constant of the motor electromagnetic force, and $r$ is the electrical resistance. Figure 1 shows a sketch of the DC motor. The available torque delivered to the coupled mechanical system is represented by $\tau$ that is the component of the available torque vector $\tau$ in the $z$-direction shown in Fig. 1.

\subsection{Motor-cart-hammer coupled system}

As described in the introduction, the system is composed by a cart whose movement is driven by the DC motor, and by a hammer that is embarked into the cart. The motor is coupled to the cart through a pin that slides into a slot machined in an acrylic plate that is attached to the cart, as shown in Fig. 2. The off-center pin is fixed on the disc at distance $\Delta$ of the motor shaft, so that the motor rotational motion is transformed into a cart horizontal movement. To model the coupling between the motor and the mechanical system, the 
motor shaft is assumed to be rigid. Thus, the available torque vector to the coupled mechanical system, $\boldsymbol{\tau}$, can be written as

$$
\boldsymbol{\tau}(t)=\boldsymbol{\Delta}(t) \times \mathbf{f}(t),
$$

where $\boldsymbol{\Delta}=(\Delta \cos \alpha(t), \Delta \sin \alpha(t), 0)$ is the vector related to the eccentricity of the pin, and where $\mathbf{f}$ is the coupling force between the DC motor and the cart. Assuming that there is a viscous friction between the pin and the slot, the vector $\mathbf{f}$ has two components: the horizontal force that the DC motor exerts in the cart, $f_{x}$, and the vertical force, $f_{y}$, induced by the viscous friction. The available torque $\tau$ and vertical force $f_{y}$ are written as

$$
\begin{gathered}
\tau(t)=f_{y}(t) \Delta \cos \alpha(t)-f_{x}(t) \Delta \sin \alpha(t), \\
f_{y}(t)=c_{p i n} \Delta \dot{\alpha}(t) \cos \alpha(t),
\end{gathered}
$$

where $c_{\text {pin }}$ is the viscous friction. The embarked hammer is modeled as a rigid body of mass $m_{h}$ and its relative displacement is $h$ with respect to the cart. In the adopted model, the constitutive equation of the spring component between the hammer and the cart is written as $f_{s}(t)=k_{h 1} h(t)+k_{h 3} h(t)^{3}$. The rate of nonlinearity of the hammer stiffness is defined as $r_{h}=k_{h 3} / k_{h 1}$. We introduce the natural frequency, $\omega_{h}$, of the hammer suspended to the linear spring with constant $k_{h 1}$ such that $\omega_{h}=\sqrt{k_{h 1} / m_{h}}$. The horizontal cart displacement is represented by $x$. Due to constraints, the cart is not allowed to move in the vertical direction. The spring-damper element modeling the medium on which the impacts occur, is constituted of a linear spring with stiffness coefficient $k_{i}$ and a damper with damping coefficient $c_{i}$. The equations of the cart-hammer-barrier system are

$$
\begin{gathered}
\ddot{x}(t)\left(m_{c}+m_{h}\right)+\ddot{h}(t) m_{h}+c_{\text {ext }} \dot{x}(t)=-f_{\text {imp }}(t)+f_{x}(t), \\
\ddot{x}(t) m_{h}+\ddot{h}(t) m_{h}+c_{i n t} \dot{h}+k_{h 1} h(t)+k_{h 3} h^{3}(t)=-f_{\text {imp }}(t),
\end{gathered}
$$

where, $c_{e x t}$ is the viscous friction coefficient between the cart and the rail and $c_{i n t}=2 \varsigma_{i n t} \sqrt{m_{h} k_{h 1}}$ is the viscous friction coefficient between the cart and the hammer ( $\varsigma_{i n t}$ is the damping ratio). The term $f_{x}$ is the horizontal coupling force between the DC motor and the cart, and $f_{\text {imp }}$ is the impact force between the hammer and the barrier, which is written as

$$
f_{\mathrm{imp}}(t)=-\phi(t)\left(k_{i}(x(t)+h(t)+g)+c_{i}(\dot{x}(t)+\dot{h}(t))\right),
$$


where

$$
\phi(t)= \begin{cases}1, & \text { if } x(t)+h(t)+g<0 \text { and } \dot{h}(t)+\dot{x}(t)<0, \\ 0, & \text { in all other cases }\end{cases}
$$

in which $g$ is defined as the horizontal distance from the hammer (when $\alpha=\pi / 2 \mathrm{rad})$ to the equilibrium position of the barrier. In the model defined by Eq. (8), an impact starts when $x(t)+h(t)$ is negative and equal to $-g$ and, $\dot{h}(t)-\dot{x}(t)<0$. During an impact, the action of the barrier on the hammer stops as soon as the total velocity $\dot{h}(t)+\dot{x}(t)$ becomes positive (the return of the hammer). Due to the system geometry, $x(t)$ and $\alpha(t)$ are related by the following constraint

$$
x(t)=\Delta \cos (\alpha(t)) .
$$

Substituting Eqs. (3) to (9) into Eq. (1), we obtain the initial value problem for the motor-cart-hammer-barrier coupled system that is written as follows. Given a constant source voltage $\nu$, find $(\alpha, c, h)$ such that, for all $t>0$,

$$
\begin{gathered}
l \dot{c}(t)+r c(t)+k_{e} \dot{\alpha}=\nu, \\
\ddot{\alpha}(t)\left(j_{m}+\left(m_{c}+m_{h}\right) \Delta^{2} \sin (\alpha(t))^{2}\right)-\ddot{h}(t)\left(m_{h} \Delta \sin (\alpha(t))\right)-k_{e} c(t) \\
+\dot{\alpha}(t)\left(b_{m}+\dot{\alpha}(t)\left(m_{c}+m_{h}\right) \Delta^{2} \cos (\alpha(t)) \sin (\alpha(t))\right. \\
\left.+c_{p i n} \Delta^{2} \cos (\alpha(t))^{2}-c_{e x t} \Delta^{2} \sin (\alpha(t))^{2}\right) \\
=\phi\left(k_{i}(\Delta \cos (\alpha(t))+h+g)+c_{i}(-d \dot{\alpha}(t) \sin (\alpha(t))+\dot{h}(t))\right) \Delta \sin (\alpha(t)), \\
\ddot{h}(t) m_{h}-\ddot{\alpha}(t)\left(m_{h} \Delta \sin (\alpha(t))\right)-\dot{\alpha}(t)\left(m_{h} \Delta \dot{\alpha}(t) \cos (\alpha(t))\right) \\
+\dot{h}(t) c_{i n t}+k_{h 1} h(t)+k_{h 3} h^{3}(t) \\
=\phi(t)\left(k_{i}(\Delta \cos (\alpha(t))+h+g)+c_{i}(-\Delta \dot{\alpha}(t) \sin (\alpha(t))+\dot{h}(t))\right)
\end{gathered}
$$

where

$\phi(t)= \begin{cases}1, & \text { if } \Delta \cos \alpha(t)+h(t)+g<0 \text { and } \dot{h}(t)-\Delta \dot{\alpha}(t) \cos (\alpha(t))<0 \\ 0, & \text { in all other cases }\end{cases}$

with the initial conditions,

$$
\alpha(0)=0 \quad, \quad \dot{\alpha}(0)=0 \quad, \quad c(0)=\frac{\nu}{r} \quad, \quad h(0)=0 \quad, \quad \dot{h}(0)=0 .
$$




\section{Measure of the system performance}

At time $t$, the electric power introduced by the electrical grid in the motor is

$$
\pi_{\text {in }}(t)=\nu c(t)
$$

Let $t_{b}^{j}$ and $t_{e}^{j}$ be the instants of begin and end of the $j$-th impact, such that for all $t$ belonging to $\left[t_{b}^{j}, t_{e}^{j}\right]$, we have $\dot{x}(t)+\dot{h}(t)<0$. At time $t$, the impact power, $\pi_{\text {imp }}^{j}(t)$, is then written as

$$
\pi_{\mathrm{imp}}^{j}(t)=k_{i}(x(t)+h(t))(\dot{x}(t)+\dot{h}(t)), \quad t_{b}^{j} \leq t \leq t_{e}^{j} .
$$

The time average of the impact power during the $j$-th impact, $\underline{\pi}_{\text {imp }}^{j}$, is written as

$$
\underline{\pi}_{\mathrm{imp}}^{j}=\frac{1}{t_{e}^{j}-t_{b}^{j}} \int_{t_{b}^{j}}^{t_{e}^{j}} \pi_{\mathrm{imp}}^{j}(t) d t .
$$

The sum, $\pi_{\mathrm{imp}}$, of the averages of the impact powers, which is one of the variable of interest in the design optimization problem, is written as

$$
\pi_{\mathrm{imp}}=\sum_{j=1}^{N_{\mathrm{imp}}} \underline{\pi}_{\mathrm{imp}}^{j}
$$

where $N_{\text {imp }}$ is the total number of impacts that occur during time interval $[0, T]$. The time average of the electric power consumed in this time interval is

$$
\pi_{\text {elec }}=\frac{1}{T} \int_{0}^{T} \pi_{\text {in }}(t) d t .
$$

These two variables, $\pi_{\text {imp }}$ and $\pi_{\text {elec }}$, are considered for measuring the system performance. The biggest is $\pi_{\mathrm{imp}}$ and the smaller is $\pi_{\text {elec }}$, better will be the system performance.

\section{Sensitivity analysis and choice of the design parameters}

To understand the role played by each system parameter in $\pi_{\text {imp }}$ and $\pi_{\text {elec }}$, a sensitivity analysis has been done. The objective was to determine what were the system parameters that had the biggest influence in $\pi_{\text {imp }}$ and $\pi_{\text {elec }}$, in order to define those that will be the design parameters for the robust design optimization problem. The initial value problem defined by Eqs. (10) 
to (14) has been rewritten in a dimensionless form for computation and some dimensionless parameters were defined. However, in the sensitivity analysis, these dimensionless parameters were not considered as varying parameters since they do not have an easy physical interpretation. The varying parameters used for the numerical simulations are related with the design of the cart and the embarked hammer. They are:

- $m_{c} / m_{h}$, relation between the hammer mass and the cart mass;

- $k_{h 1} / m_{h}$, relation between the linear stiffness of the spring component and hammer mass (a sort of natural frequency of the hammer);

- $g$, horizontal distance from the hammer (when $\alpha=\pi / 2 \mathrm{rad}$ ) to the equilibrium position of the barrier;

- $\Delta$, eccentricity of the pin. This parameter determines the length of the cart path.

The other parameters, related with the motor properties and viscous friction coefficients, are fixed and the values of these fixed parameters are given in Table 1 . The output responses are $\pi_{\text {imp }}$ and $\pi_{\text {elec }}$. For computation, the initial

Table 1: Values of the system parameters used in simulations.

\begin{tabular}{|cl|cl|}
\hline Parameter & Value & Parameter & Value \\
\hline$m_{c}$ & $0.50 \mathrm{Kg}$ & $\nu$ & $2.4 \mathrm{~V}$ \\
$r_{h}$ & $0.301 / \mathrm{m}^{2}$ & $r$ & $0.307 \Omega$ \\
$c_{\text {pin }}$ & $5.00 \mathrm{Ns} / \mathrm{m}$ & $l$ & $1.88 \times 10^{-4} \mathrm{H}$ \\
$c_{\text {ext }}$ & $5.00 \mathrm{Ns} / \mathrm{m}$ & $j_{m}$ & $1.21 \times 10^{-4} \mathrm{Kg} \mathrm{m}^{2}$ \\
$\varsigma_{\text {int }}$ & 0.05 & $b_{m}$ & $1.5452 \times 10^{-4} \mathrm{Nm} /(\mathrm{rad} / \mathrm{s})$ \\
$k_{i}$ & $10^{6} \mathrm{~N} / \mathrm{m}$ & $k_{e}$ & $0.0533 \mathrm{~V} /(\mathrm{rad} / \mathrm{s})$ \\
$c_{i}$ & $10^{3} \mathrm{Ns} / \mathrm{m}$ & & \\
\hline
\end{tabular}

value problem defined by Eqs. (10) to (14) has been rewritten in a dimensionless form. The main objective was to reduce the computation time. Duration is chosen as $T=10.0 \mathrm{~s}$. The 4th-order Runge-Kutta method is used for the time integration scheme for which we have implemented a varying time-step. The time-step is adapted to the state of the dynamical system according to the occurrence or the non occurrence of impacts. When the hammer is not impacting the barrier, the time step used is $10^{-4} \mathrm{~s}$, but when the hammer is 


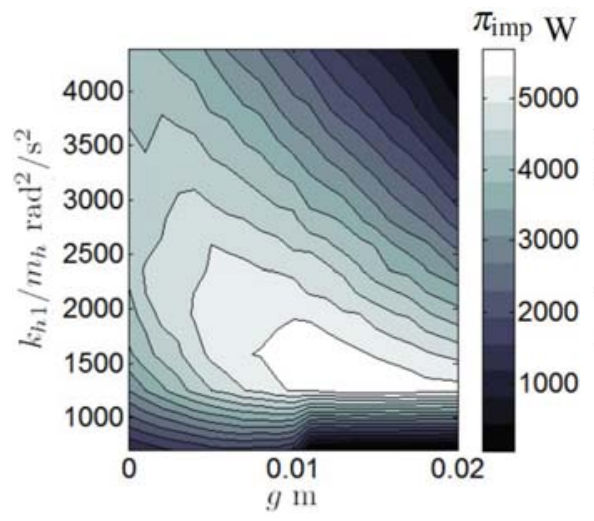

(a)

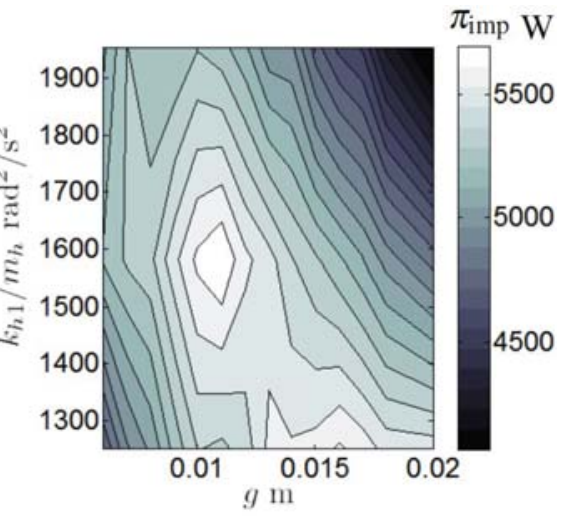

(b)

Figure 3: For the optimal values $\left(m_{c} / m_{h}\right)^{\star}$ and $\Delta^{\star}$ : (a) graph of $\pi_{\text {imp }}$ as a function of $g$ and $k_{h 1} / m_{h}$ (varying in all its range of values), (b) graph of $\pi_{\text {imp }}$ as a function of $g$ and $k_{h 1} / m_{h}$ (varying in $[0.06,0.02]$ and $[1250,1953]$ respectively).

approaching the barrier and when it is impacting it, the time step is chosen as the value $10^{-5} \mathrm{~s}$. Simulations with different values to the initial conditions, were performed. As it was verified that they do not have a significant influence in $\pi_{\text {imp }}$ and $\pi_{\text {elec }}$, in all simulations the initial conditions were taken as constant, given by Eq. (14). Concerning the sensitivity analysis, 20, 000 numerical simulations have been carried out combining the following values of the parameters: 10 values for $m_{c} / m_{h}$ selected in the interval $[0.10,2.00]$, 10 values for $k_{h 1} / m_{h}$ in $[657,4410] \mathrm{rad}^{2} / \mathrm{s}^{2}, 10$ values for $g$ in $[0,0.02] \mathrm{m}$, and 20 values for $\Delta$ in $[0.003,0.013] \mathrm{m}$. The largest value of $\pi_{\text {imp }}$, obtained with such numerical simulations, is $5,690 \mathrm{~W}$, and is reached for the following values of the parameters: $\left(m_{c} / m_{h}\right)^{\star}=0.40,\left(k_{h 1} / m_{h}\right)^{\star}=1,580 \mathrm{rad}^{2} / \mathrm{s}^{2}$, $g^{\star}=0.011 \mathrm{~m}$, and $\Delta^{\star}=0.013 \mathrm{~m}$. With these values, the average of the consumed electric power is $\pi_{\text {elec }}=3.93 \mathrm{~W}$. For $\Delta=\Delta^{\star}$ and $m_{c} / m_{h}=\left(m_{c} / m_{h}\right)^{\star}$, Fig. 3 displays $\pi_{\text {imp }}$ as a function of parameters $g$ and $k_{h 1} / m_{h}$. In Fig. 3(a), $g$ and $k_{h 1} / m_{h}$ vary in all its range of values, and in Fig. 3(b), they vary in $[0.06,0.02]$ and $[1250,1953]$ respectively. These figures show that, the optimal value of the design parameter correspond to a global maximum. The influence of each parameter in $\pi_{\text {imp }}$ and $\pi_{\text {elec }}$ can be observed through the graphs plotted in Figs. 4 to 7 . Regarding all the graphs of $\pi_{\text {imp }}$ and $\pi_{\text {elec }}$ as a function $m_{c} / m_{h}, k_{h 1} / m_{h}, g$ and $\Delta$, it can be seen that small variations on $g, k_{h 1} / m_{h}$, and $\Delta$ induce large variations for $\pi_{\text {imp }}$ and for $\pi_{\text {elec }}$, but the same 


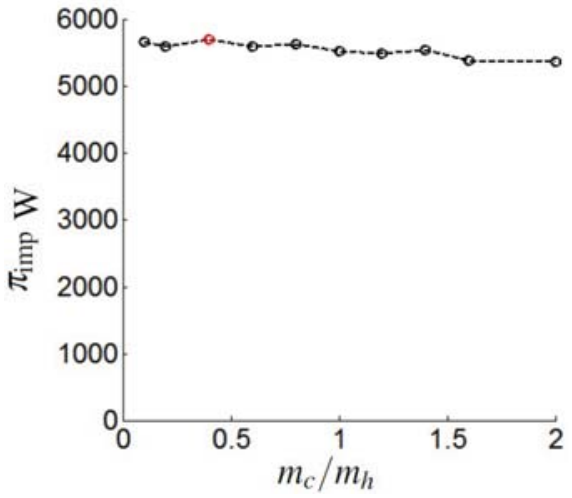

(a)

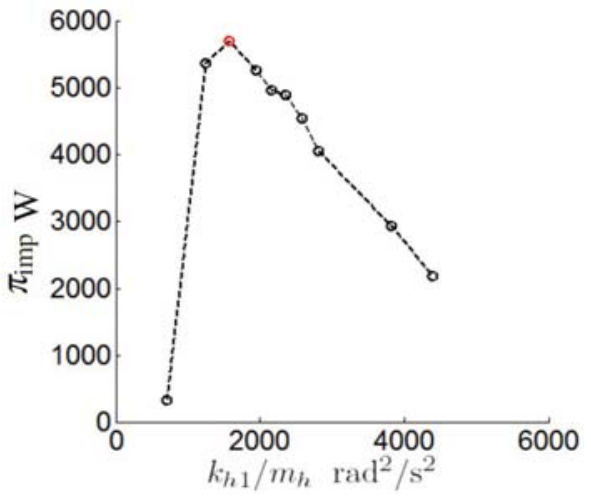

(b)

Figure 4: (a) Graph of $\pi_{\text {imp }}$ as a function of $m_{c} / m_{h}$ with $\left(k_{h 1} / m_{h}\right)^{\star}, g^{\star}$, and $\Delta^{\star}$. (b) Graph of $\pi_{\text {imp }}$ as a function of $k_{h 1} / m_{h}$ with $\left(m_{c} / m_{h}\right)^{\star}, g^{\star}$, and $\Delta^{\star}$.

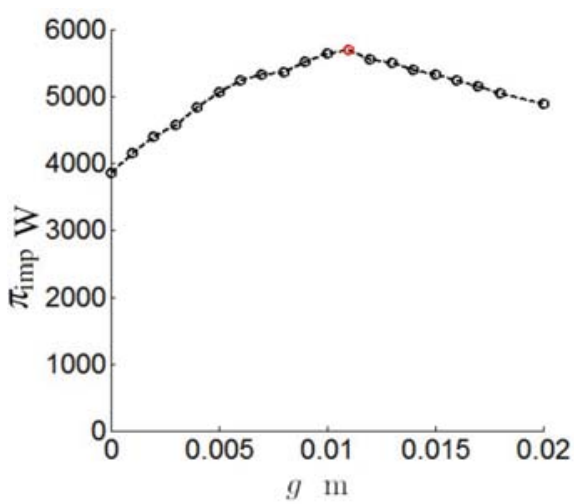

(a)

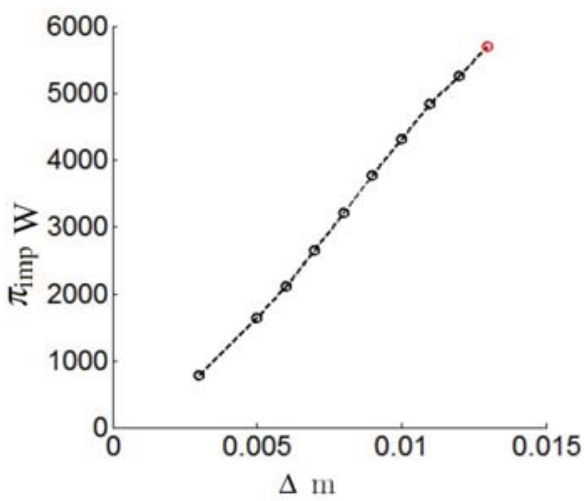

(b)

Figure 5: (a) Graph of $\pi_{\text {imp }}$ as a function of $g$ with $\left(m_{c} / m_{h}\right)^{\star},\left(k_{h 1} / m_{h}\right)^{\star}$, and $\Delta^{\star}$. (b) Graph of $\pi_{\text {imp }}$ as a function of $\Delta$ with $\left(m_{c} / m_{h}\right)^{\star},\left(k_{h 1} / m_{h}\right)^{\star}$, and $g^{\star}$. 


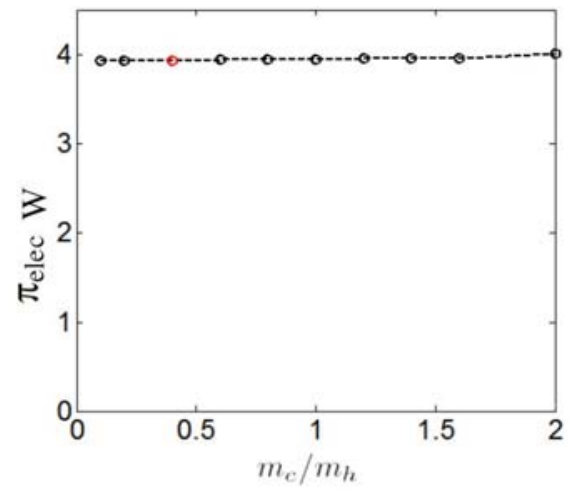

(a)

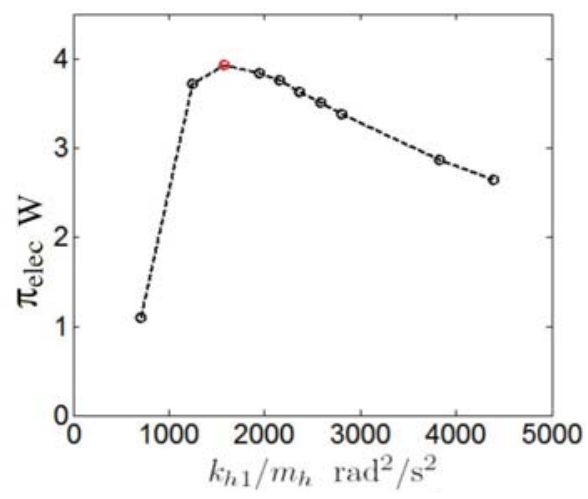

(b)

Figure 6: (a) Graph of $\pi_{\text {elec }}$ as a function of $m_{c} / m_{h}$ with $\left(k_{h 1} / m_{h}\right)^{\star}, g^{\star}$, and $\Delta^{\star}$. (b) Graph of $\pi_{\text {elec }}$ as a function of $k_{h 1} / m_{h}$ with $\left(m_{c} / m_{h}\right)^{\star}, g^{\star}$, and $\Delta^{\star}$.

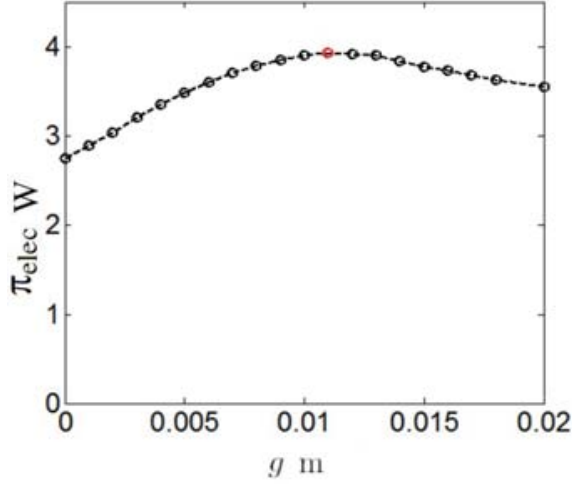

(a)

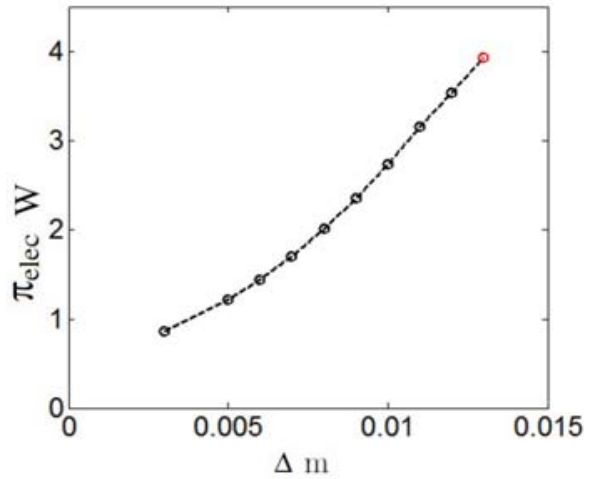

(b)

Figure 7: (a) Graph of $\pi_{\text {elec }}$ as a function of $g$ with fix $\left(m_{c} / m_{h}\right)^{\star},\left(k_{h 1} / m_{h}\right)^{\star}$, and $\Delta^{\star}$. (b) Graph of $\pi_{\text {elec }}$ as a function of $\Delta$ with fix $\left(m_{c} / m_{h}\right)^{\star},\left(k_{h 1} / m_{h}\right)^{\star}$, and $g^{\star}$. 
phenomenon does not occur with respect to the parameter $m_{c} / m_{h}$. Thus, while $\pi_{\text {imp }}$ and $\pi_{\text {elec }}$ are not very sensitive to $m_{c} / m_{h}$, they are sensitive to $k_{h 1} / m_{h}, g$ and $\Delta$. It is also seen, that two different kinds of sensitivity can be distinguished among these three parameters. For parameters $k_{h 1} / m_{h}$ and $g$, it can be seen that $\pi_{\text {imp }}$ and $\pi_{\text {elec }}$ reach their maxima when $k_{h 1} / m_{h}$ and $g$ are equal to $1,580 \mathrm{rad}^{2} / \mathrm{s}^{2}$ and $0.011 \mathrm{~m}$ respectively. For parameter $\Delta$ varying in its range of values, Figs. $5(\mathrm{~b})$ and $7(\mathrm{~b})$ show that the highest is $\Delta$,

the highest are $\pi_{\text {imp }}$ and $\pi_{\text {elec }}$. It has been considered as not necessarily to verify the behavior of $\pi_{\text {imp }}$ and $\pi_{\text {elec }}$ for a larger range of $\Delta$ because the value $\Delta=0.013 \mathrm{~m}$ is already sufficiently large when compared with the system dimensions and the motor properties. It should be noted that if parameter $\Delta$ were increased more then, the nonlinearities would increase more, that is not the objective of the analysis. Considering that $m_{c} / m_{h}$ does not have a significant influence in $\pi_{\text {imp }}$ and $\pi_{\text {elec}}$, and considering that the sensitivity of the parameter $\Delta$ is easily predictable, these two parameters will not be considered as design parameters in the robust design optimization problem. Only parameters $g$ and $k_{h 1} / m_{h}$ will thus be considered as design parameters.

\section{Construction of the probability model}

As explained in the introduction, this paper deals with the robust design of the electro-mechanical system in presence of uncertainties in the computational model. The three parameters that are assumed to be uncertain are $k_{h 1}, k_{i}$ and $c_{i}$, which are modeled by the independent random variables $K_{h 1}$, $K_{i}$ and $C_{i}$. The probability distribution of each one is constructed using the Maximum Entropy Principle [19, 20]. This principle allows the probability distribution of a random variable to be constructed using only the available information, avoiding the use of any additional information that introduces a bias on the estimation of the probability distribution. If a large amount of experimental data are available, then the nonparametric statistics can be used. If there are no available experimental data, or if there are only a few experimental data, then the Maximum Entropy from Information Theory is the most efficient tool for constructing a prior probability model for which its hyperparameters are used as sensitivity parameters if there are no experimental data, or, are estimated in using the parametric statistics if a few experimental data are available. The Maximum Entropy Principle states: out of all probability distributions consistent with a given set of available information, choose the one that has maximum uncertainty (the Shannon 
measure of entropy). The available information of the random variables is defined as

1. $K_{h 1}, K_{i}$ and $C_{i}$ are positive-valued random variables,

2. the mean values are known: $E\left\{K_{i}\right\}=\underline{K}_{i}, E\left\{C_{i}\right\}=\underline{C}_{i}$ and $E\left\{K_{h 1}\right\}=$ $\underline{K}_{h 1}$,

3. in order that the response of the dynamical system be a second-order stochastic process, we impose the following conditions: $\left\|E\left\{\log K_{i}\right\}\right\|<$ $\infty,\left\|E\left\{\log C_{i}\right\}\right\|<\infty$ and $\left\|E\left\{\log K_{h 1}\right\}\right\|<\infty$.

Thus, the Maximum Entropy Principle for each random variable $K_{i}, C_{i}$, and $K_{h 1}$, yields a Gamma distribution (see [21]),

$$
p(a)=\mathbb{1}_{[0,+\infty)}(a) \frac{1}{\mu}\left(\frac{1}{\delta^{2}}\right)^{\frac{1}{\delta^{2}}} \frac{1}{\Gamma\left(1 / \delta^{2}\right)}\left(\frac{a}{\mu}\right)^{\frac{1}{\delta^{2}}-1} \exp \left(\frac{a}{\delta^{2} \mu}\right),
$$

where $\mathbb{1}_{[0,+\infty)}(a)$ is an indicator function that is equal to 1 for $a \in[0,+\infty)$ and 0 otherwise, and where

- $\Gamma$ is the Gamma function: $\Gamma(b)=\int_{0}^{\infty} t^{b-1} \exp (-t) \mathrm{d} t$;

- $\delta=\frac{\sigma}{\mu}$ is the coefficient variation of the random variable, $\mu$ is its mean value representing $\underline{K}_{i}, \underline{C}_{i}$, or $\underline{K}_{h 1}$, and $\sigma$ is its standard deviation.

\section{Robust design optimization problem}

In order to formulate the robust design problem, the set of all the system parameters is divided into three subsets. The first subset is the family of the fixed parameters that is represented by the vector $\mathbf{p}_{\text {fix }}=\{\nu$, $\left.l, r, j_{m}, k_{e}, b_{m}, c_{p i n}, c_{e x t}, \varsigma_{i n t}, r_{h}, m_{c}, m_{h}, \Delta\right\}$. The second one is the family of the design parameters that is represented by the vector $\mathbf{p}_{\text {des }}=$ $\left\{\underline{K}_{h 1} / m_{h}, g\right\}$. The third one is the family of the uncertain parameters that is represented by the random vector $\mathbf{P}_{\text {unc }}=\left\{K_{i}, C_{i}, K_{h 1}\right\}$. Since $\mathbf{P}_{\text {unc }}$ is a random vector, the outputs of the electro-mechanical system are stochastic processes and, consequently, $\pi_{\text {imp }}\left(\mathbf{p}_{\text {des }}, \mathbf{p}_{\text {unc }}\right)$ and $\pi_{\text {elec }}\left(\mathbf{p}_{\text {des }}, \mathbf{p}_{\text {unc }}\right)$, become random variables $\Pi_{\text {imp }}\left(\mathbf{p}_{\text {des }}\right)=\pi_{\text {imp }}\left(\mathbf{p}_{\text {des }}, \mathbf{P}_{\text {unc }}\right)$ and $\Pi_{\text {elec }}\left(\mathbf{p}_{\text {des }}\right)=$ $\pi_{\text {elec }}\left(\mathbf{p}_{\text {des }}, \mathbf{P}_{\text {unc }}\right)$. The cost function of the robust design optimization problem is defined by

$$
J\left(\mathbf{p}_{\text {des }}\right)=E\left\{\Pi_{\text {imp }}\left(\mathbf{p}_{\text {des }}\right)\right\} .
$$


The robust design optimization problem is written as

$$
\mathbf{p}_{\text {des }}^{\text {opt }}=\underset{\mathbf{p}_{\text {des }} \in C_{a d}}{\arg \max } J\left(\mathbf{p}_{\text {des }}\right),
$$

in which $\mathcal{C}_{a d}=\left\{\mathbf{p}_{\text {des }} \in \mathcal{P}_{\text {des }} ; E\left\{\Pi_{\text {elec }}\left(\mathbf{p}_{\text {des }}\right)\right\} \leq c_{\text {elec }}\right\}$, where $\mathcal{P}_{\text {des }}$ is the admissible set of the values of $\mathbf{p}_{\text {des }}$, and where $c_{\text {elec }}$ is an upper bound.

\section{Results of the robust optimization problem}

The hyperparameters $\delta_{K_{i}}$ and $\delta_{C_{i}}$, which control the level of uncertainties for $K_{i}$ and $C_{i}$ are fixed to 0.1 . The robust design optimization problem is then solved for three levels of uncertainties for $K_{h 1}$, defined by the following values of the hyperparameters $\delta_{K_{h 1}}=0, \delta_{K_{h 1}}=0.1$, and $\delta_{K_{h 1}}=0.4$. The optimization problem is also considered whitout uncertainties in the systems parameters, that is, the deterministic case $\left(\delta_{K_{h 1}}=\delta_{K_{i}}=\delta_{C_{i}}=0\right)$. For $\mathbf{p}_{\text {des }} \in \mathcal{C}_{a d}$, the cost function is estimated by the Monte Carlo simulation method using 100 independent realizations of random vector $\mathbf{P}_{\text {unc }}$ following its probability distribution. The optimization problem (defined by Eq. (22)) is solved using the trial method for which the admissible set $\mathcal{C}_{a d}$ is meshed as follows: for $\underline{K}_{h 1} / m_{h}, 13$ values are nonuniformly selected in the interval [703, 3830], and for $g, 20$ nonuniform values in [0,0.038]. Thus, 26, 000 numerical simulations have been carried out to solve optimization problem for each level of uncertainties. For computation, the initial value problem, defined by Eqs. (10) to (14) and rewritten in a dimensionless form, is solved using the time-integration scheme described in Section 4 with duration $T=$ $10.0 \mathrm{~s}$. The values of the fixed parameters are $m_{c}=0.3 \mathrm{Kg}, m_{h}=0.5 \mathrm{Kg}$, $\Delta=0.01 \mathrm{~m}$, and the others are given in Table 1 . Upper bound $c_{\text {elec }}$ is fixed to the value $6.00 \mathrm{~W}$. For the deterministic case, the components of the optimal solution $\mathbf{p}_{\text {des }}^{\text {opt }}$ are $\left(\underline{K}_{h 1} / m_{h}\right)^{\text {opt }}=1,580 \mathrm{rad}^{2} / \mathrm{s}^{2}$ and $g^{\mathrm{opt}}=0.011 \mathrm{~m}$. For case with uncertainties, for which $\delta_{K_{i}}$ is fixed to 0.1 , and $\delta_{C_{i}}$ to 0.1 , we obtain, for $\delta_{K_{h 1}}=0,\left(\underline{K}_{h 1} / m_{h}\right)^{\text {opt }}=957 \mathrm{rad}^{2} / \mathrm{s}^{2}$ and $g^{\text {opt }}=0.018 \mathrm{~m}$, for $\delta_{K_{h 1}}=0.1,\left(\underline{K}_{h 1} / m_{h}\right)^{\mathrm{opt}}=1,950 \mathrm{rad}^{2} / \mathrm{s}^{2}$ and $g^{\text {opt }}=0.008 \mathrm{~m}$, and for $\delta_{K_{h 1}}=0.4,\left(\underline{K}_{h 1} / m_{h}\right)^{\text {opt }}=2,360 \mathrm{rad}^{2} / \mathrm{s}^{2}$ and $g^{\text {opt }}=0.008 \mathrm{~m}$. Figures 8 and 9 display the graphs of the cost function defined by Eq. (21) as a function of the design parameter for these four cases. These figures show that, for each case, the optimal value of the design parameter correspond to a global maximum in $\mathcal{C}_{a d}$. The role played by uncertainties on the optimal values of the design parameters can be analyzed through Figs. 10 and 11, which display the graphs 


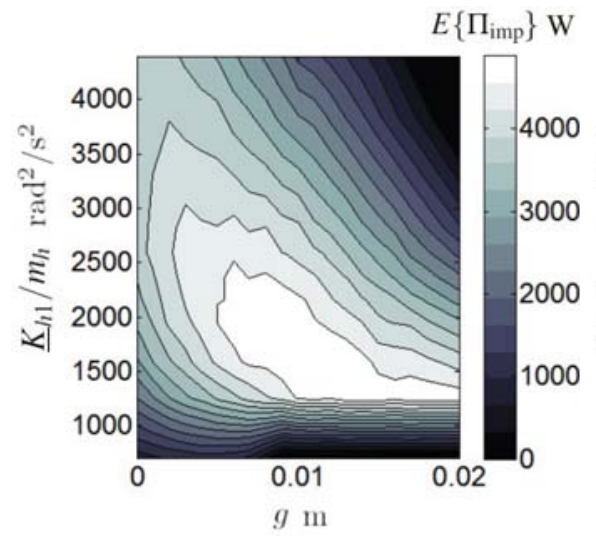

(a)

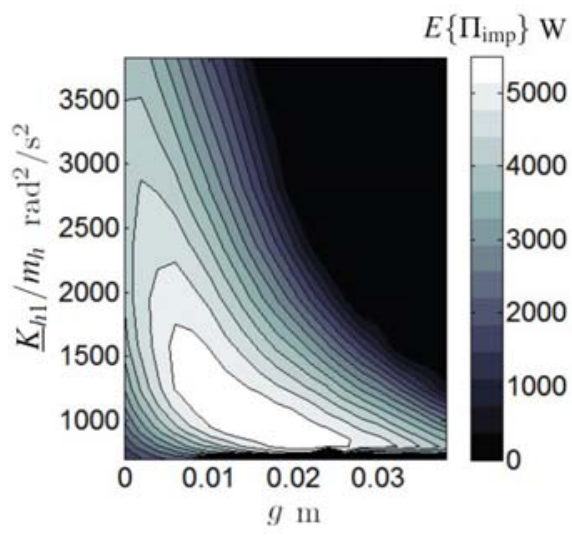

(b)

Figure 8: (a) Cost function as function of the design parameters for the deterministic case. (b) Cost function as function of the design parameters for the case in which $\delta_{K_{i}}=\delta_{C_{i}}=0.1$ and $\delta_{K_{h 1}}=0$.

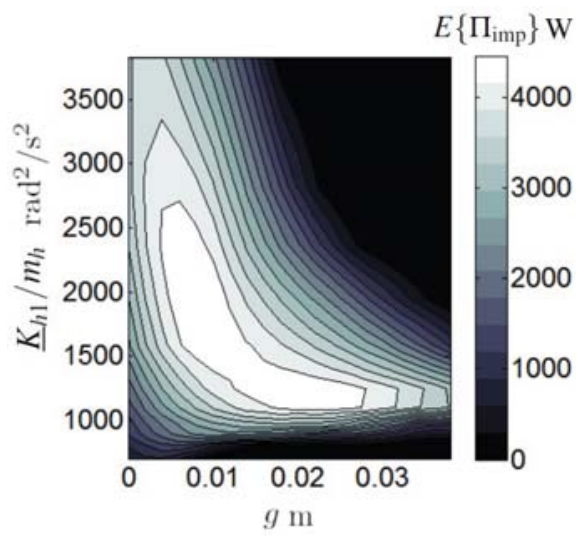

(a)

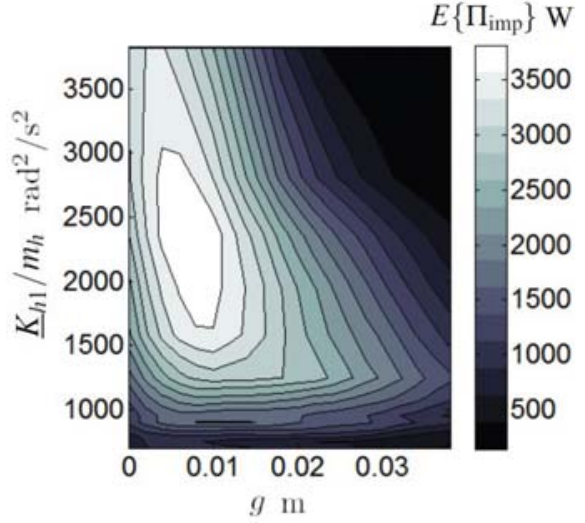

(b)

Figure 9: (a) Cost function as function of the design parameters for the case in which $\delta_{K_{i}}=\delta_{C_{i}}=\delta_{K_{h 1}}=0.1$. (b) Cost function as function of the design parameters for the case in which $\delta_{K_{i}}=\delta_{C_{i}}=0.1$ and $\delta_{K_{h 1}}=0.4$. 


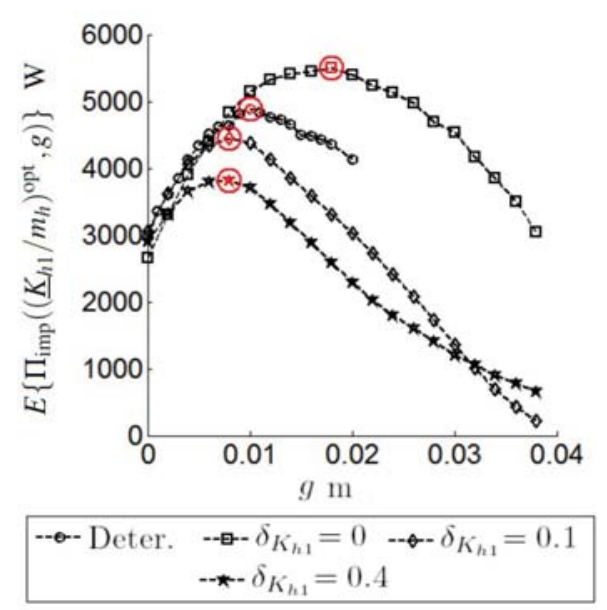

(a)

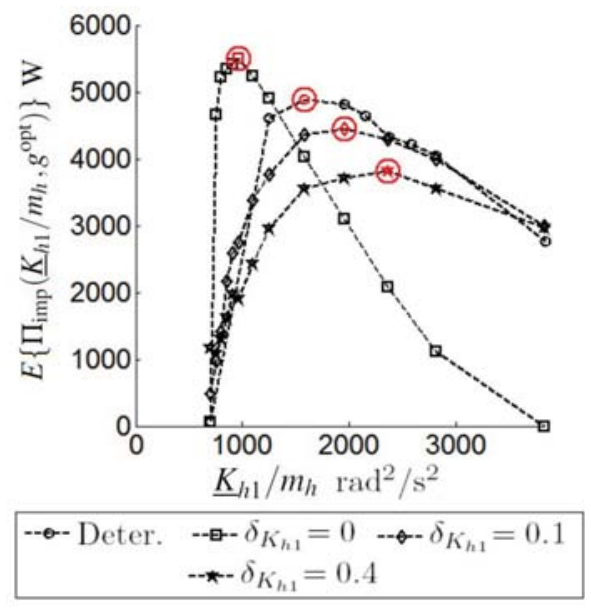

(b)

Figure 10: (a) Cost function as function of $g$ with $\left(\underline{K}_{h 1} / m_{h}\right)^{\text {opt }}$. (b) Cost function as function of $\underline{K}_{h 1} / m_{h}$ with $g^{\text {opt }}$. In both graphs, the $E\left\{\Pi_{\text {imp }}\left(\mathbf{p}_{\text {des }}^{\text {opt }}\right)\right\}$ is highlighted for each level of uncertainties with markers.

$g \mapsto E\left\{\Pi_{\text {imp }}\left(\left(\underline{K}_{h 1} / m_{h}\right)^{\text {opt }}, g\right)\right\}, \underline{K}_{h 1} / m_{h} \mapsto E\left\{\Pi_{\text {imp }}\left(\underline{K}_{h 1} / m_{h}, g^{\text {opt }}\right)\right\}, g \mapsto$ $E\left\{\Pi_{\text {elec }}\left(\left(\underline{K}_{h 1} / m_{h}\right)^{\text {opt }}, g\right)\right\}$, and $\underline{K}_{h 1} / m_{h} \mapsto E\left\{\Pi_{\text {elec }}\left(\underline{K}_{h 1} / m_{h}, g^{\text {opt }}\right)\right\}$. These figures show that the optimal design point strongly depends on the level of uncertainties. In particular, it can be deduced that the mean value of the electric power increases with the increase of the gap. The robustness of the optimal design point, $\mathbf{p}_{\mathrm{des}}^{\mathrm{opt}}$, can be analyzed in studying the evolution of the coefficient variation, $\delta_{\Pi_{\mathrm{imp}}}\left(\mathbf{p}_{\mathrm{des}}^{\mathrm{opt}}\right)$, of random variable $\Pi_{\mathrm{imp}}\left(\mathbf{p}_{\mathrm{des}}^{\mathrm{opt}}\right)$ as a function of the uncertainty level. However, in order to better analyze the sensitivity of the responses with respect to the uncertainty level, we have constructed Fig. 12 that displays the graphs $\left.g \mapsto \delta_{\Pi_{\mathrm{imp}}}\left(\underline{K}_{h 1} / m_{h}\right)^{\text {opt }}, g\right)$ and $\underline{K}_{h 1} / m_{h} \mapsto \delta_{\Pi_{\text {imp }}}\left(\underline{K}_{h 1} / m_{h}, g^{\text {opt }}\right)$. For each level of uncertainties, it can be seen that the value $\delta_{\Pi_{\mathrm{imp}}}\left(\mathbf{p}_{\mathrm{des}}^{\mathrm{opt}}\right)$ occurs in a region for which the two following functions $g \mapsto \delta_{\Pi_{\mathrm{imp}}}\left(\left(\underline{K}_{h 1} / m_{h}\right)^{\mathrm{opt}}, g\right)$ and $\underline{K}_{h 1} / m_{h} \mapsto \delta_{\Pi_{\mathrm{imp}}}\left(\underline{K}_{h 1} / m_{h}, g^{\mathrm{opt}}\right)$ are minima. This means the optimal design point is robust with respect to uncertainties.

\section{Conclusions}

In this paper, the formulation and the solution of a robust design optimization problem have been presented for a nonlinear electro-mechanical 


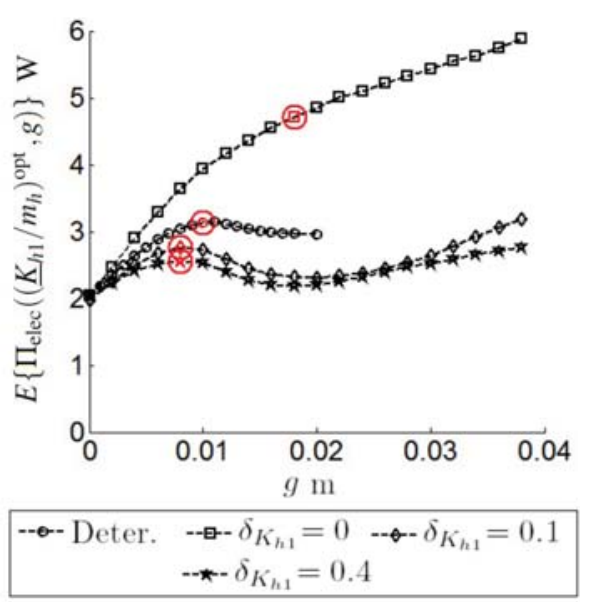

(a)

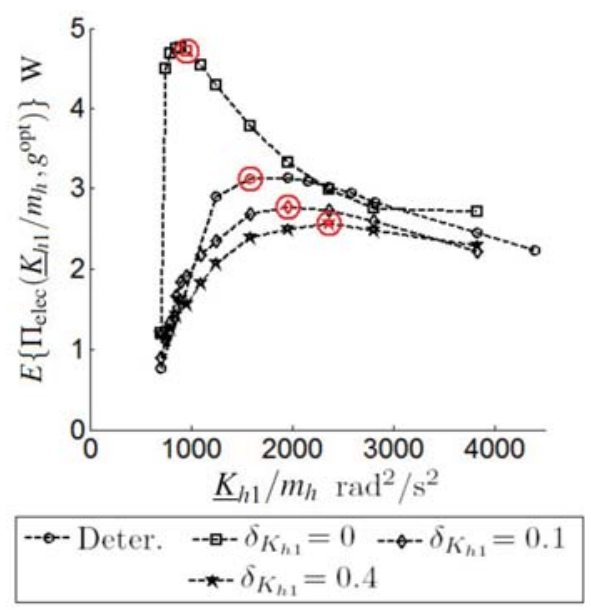

(b)

Figure 11: (a) Mean value of the time average of electric power as function of $g$ with $\left(\underline{K}_{h 1} / m_{h}\right)^{\text {opt }}$. (b) Mean value of the time average of electric power as function of $\underline{K}_{h 1} / m_{h}$ with $g^{\text {opt }}$. In both graphs, the $E\left\{\Pi_{\text {elec }}\left(\mathbf{p}_{\text {des }}^{\text {opt }}\right)\right\}$ is highlighted for each level of uncertainties with markers.

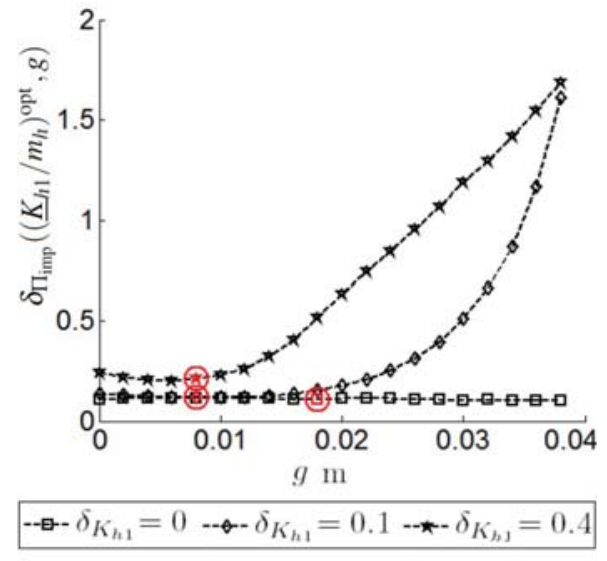

(a)

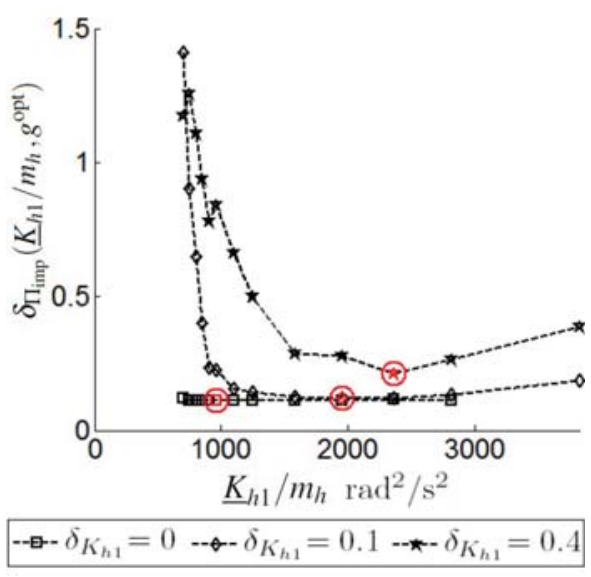

(b)

Figure 12: (a) Coefficient variation of $\Pi_{i m p}$ as function of $g$ with $\left(\underline{K}_{h 1} / m_{h}\right)^{\text {opt }}$ (b) Coefficient variation of $\Pi_{\mathrm{imp}}$ as function of $\underline{K}_{h 1} / m_{h}$ with $g^{\mathrm{opt}}$. In both graphs, the $\delta_{\Pi_{\mathrm{imp}}}\left(\mathbf{p}_{\mathrm{des}}^{\mathrm{opt}}\right)$ is highlighted for each level of uncertainties with markers. 
vibro-impact system in presence of uncertainties in the computational model. Since this nonlinear electro-mechanical system is devoted to the vibro-impact optimization, the time responses exhibit numerous shocks that have to be identified with accuracy, and consequently, a very small time step is required. We have thus chosen an explicit time-integration scheme and not an implicit one. Nevertheless, due to the presence of low-frequency contributions in the time responses, a long time duration is required, which will imply a huge number of integration time step if the time step were chosen constant. This is the reason why we have implemented an adaptive integration time step. It was one of the difficulties encountered for the solver implementation. The use a varying time-step integration scheme was not the only strategy adopted to reduce the computation time. The initial value problem has been rewritten in a dimensionless form, which reduced the computation time of each simulation from 8 minutes to 5 minutes on average. Furthermore, a cluster with 20 computers has been used to to parallelize the simulations carried out in the sensitivity analysis and in the optimization problem. Observing the results of numerical integration, as time histories and phase diagrams, some interesting phenomena were verified, as for example bifurcation. Bifurcation is a typical nonlinear phenomena, and it is frequently discussed in many works (see for instance [22]). In the present paper, it appears because depending on the values of the system parameters, the system response will have the occurrence or the non occurrence of impacts. But this topic is an ongoing research that will be object of a future work. The construction of the solution for the design optimization problem, has been prepared by carrying out a sensitivity analysis with respect all the possible design parameters. This pre-analysis has allowed for reducing the number of design parameters to two parameters. Consequently, a random search algorithm or a genetic algorithm was not necessary, and we have thus used a trail method. It should be noted that in the framework of a robust analysis formulated in the context of the probability theory, and taking into account the types of nonlinearities in the dynamical system, the Monte Carlo numerical simulation method has been used, and this introduces a significant increase of the numerical cost. The design optimization problem of the dynamical system without uncertainties yields an optimal design point that differs from the nominal values, and which can not be determined, a priori, without solving the design optimization problem. In addition, the robust analysis that has been presented demonstrates the interest that there is to take into account the uncertainties in the computational model. The optimal design point that 
has been identified in the robust design framework significantly differs from design point obtained with the computational model without uncertainties. For this electro-mechanical system, it has been seen that, the minimum value of the dispersion of the random output occurs in the region of the optimal design parameters, which means that the optimal design point is robust with respect to uncertainties.

\section{Acknowledgements}

The authors acknowledge the support given by FAPERJ, CNPq and CAPES.

\section{References}

[1] S. Zhankui, K. Sun, Nonlinear and chaos control of a micro-electromechanical system by using second-order fast terminal sliding mode control, Communications in Nonlinear Science and Numerical Simulation 18 (2013) 2540-2548.

[2] H. Sadeghian, G. Rezazadeh, Comparison of generalized differential quadrature and galerkin methods for the analysis of micro-electromechanical coupled systems, Communications in Nonlinear Science and Numerical Simulation 14 (2009) 2807-2816.

[3] H. Lee, I. Incheon, C. Cho, S. Chang, Design and analysis of electromechanical characteristics of micromachined stainless steel pressure sensor, in: Proc. of the 5th IEEE Sensors Conference, IEEE Sensors, IEEE, Daegu, South Korea, 2006, pp. 659-674.

[4] Y. Rocard, Dynamique Générale des Vibrations, Masson et Cie., Éditeurs, Paris, France, 1943.

[5] V. O. Kononenko, Vibrating Systems with a Limited Power Supply, London Iliffe Books LTD, England, 1969.

[6] A. H. Nayfeh, D. T. Mook, Nonlinear Oscillations, John Wiley and Sons, USA, 1979.

[7] A. Fidlin, Nonlinear Oscillations in Mechanical Engineering, Springer, Netherlands, 2006. 
[8] P. Hagedorn, Non-linear Oscillations, 2nd Edition, Clarendon Press, Oxford, 1988.

[9] R. M. Evan-Iwanowski, Resonance oscillations in mechanical systems, Elsevier Publ. Co., Amsterdam, 1976.

[10] M. Cartmell, Introduction to Linear, Parametric and Nonlinear Vibrations, Vol. 260, Springer, 1990.

[11] R. Lima, R. Sampaio, Stochastic analysis of an electromechanical coupled system with embarked mass, Mecânica Computacional XXXI (2012) 2709-2733, http://www.cimec.org.ar/ojs/index.php/mc/article/view/4216/4142.

[12] G. Luo, Y. Zhang, J. Xie, J. Zhang, Periodic-impact motions and bifurcations of vibro-impact systems near 1:4 strong resonance point, Communications in Nonlinear Science and Numerical Simulation 13 (2008) $1002-1014$.

[13] V. Ostasevicius, R. Gaidys, R. Dauksevicius, Numerical analysis of dynamic effects of a nonlinear vibro-impact process for enhancing the reliability of contact-type mems devices, Sensors 9 (2009) 10201-10216.

[14] X. Yue, W. Xu, L. Wang, Global analysis of boundary and interior crises in an elastic impact oscillator, Communications in Nonlinear Science and Numerical Simulation 18 (2013) 3567-3574.

[15] R. Ibrahim, Vibro-Impact Dynamics: Modeling, Mapping and Applications, Springer, Berlin, 2009.

[16] F. Nucera, A. Vakakis, D. McFarland, L. Bergman, G. Kerschen, Targeted energy transfers in vibro-impact oscillators for seismic mitigation, Nonlinear Dynamics 50 (2007) 651-677.

[17] R. Sampaio, C. Soize, On measures of non-linearity effects for uncertain dynamical systems - application to a vibro-impact system, Journal of Sound and Vibration 303 (3-5) (2007) 659-674.

[18] D. Karnopp, D. Margolis, R. Rosenberg, System Dynamics: Modeling and Simulation of Mechatronic Systems, John Wiley and Sons, 4th edition, New-York, USA, 2006. 
[19] E. Jaynes, Information theory and statistical mechanics, The Physical Review 106 (4) (1957) 620-630.

[20] C. Shannon, A mathematical theory of communication, Bell System Tech. 27 (1948) 379-423 and 623-659.

[21] C. Soize, Maximum entropy approach for modeling random uncertainties in transient elastodynamics, Journal of the Acoustical Society of America 109 (5) (2001) 1979-1996.

[22] R. Ragulskis, K. Ragulskis, The effect of dynamical self-orientation and its applicability for identification of natural frequencies, Nonlinear Dynamics 50 (2007) 61-71. 